\title{
Power Optimal Scheduling for Guaranteed Throughput in Multi-access Fading Channels
}

\author{
Prasanna Chaporkar, Kimmo Kansanen, Ralf R. Müller \\ Institute of Electronics and Telecommunications \\ Norwegian University of Science and Technology \\ Trondheim, Norway
}

\begin{abstract}
A power optimal scheduling algorithm that guarantees desired throughput and bounded delay to each user is developed for fading multi-access multi-band systems. The optimization is over the joint space of all rate allocation and coding strategies. The proposed scheduling assigns rates on each band based only on the current system state, and subsequently uses optimal multi-user signaling to achieve these rates. The scheduling is computationally simple, and hence scalable. Due to uplink-downlink duality, all the results extend in straightforward fashion to the broadcast channels.
\end{abstract}

\section{Index Terms}

Power minimization, scheduling, stability, convex optimization, super-position encoding and successive decoding. 


\section{INTRODUCTION}

We consider a multi-access fading channel with $N$ users and a single access point. Each user $k$ requires certain long term rate (throughput) guarantee $a_{k}$. Our aim is to design a scheduling strategy that arbitrates, in every slot, the instantaneous rate assignment to each user and coding strategy to realize the assigned rates depending on the current fading states so that the throughput requirement for each user is fulfilled and the total power expenditure is minimized.

In their seminal work, Tse and Hanly have characterized so called throughput capacity and delaylimited capacity of the multi-access fading channel with Gaussian noise [1], [2]. The throughput capacity region quantifies the achievable rate region with average power constraint for ergodic fading. For the delay limited capacity, each user must be given the required rate irrespective of its fading states in every slot (strict delay of one slot). The aim here is to obtain a coding and power allocation scheme to minimize the energy.

The notion of throughput capacity leads to schemes that take advantage of users' differential channel qualities. Specifically, it is known that the sum throughput in the system is maximized by letting only one user with the best channel transmit. Schemes that take current channel states into account while making scheduling decisions are referred to as "Opportunistic Scheduling" and may result in unfair rate allocation if the fading statistics are not symmetric which is typical in wireless systems. To alleviate this limitation, several opportunistic scheduling schemes with fairness constraints have been designed [3], [4]. Among them, Proportional Fair Scheduling (PFS) has many desirable properties including provable fairness guarantees and suitability for on-line implementation, i.e., without prior knowledge of channel statistics [5]. But, PFS does not guarantee the required throughput to users.

Unlike opportunistic scheduling schemes, the delay-limited schemes guarantee the required throughput to every user. Specifically, super-position encoding and successive decoding is shown to minimize power for achieving the required throughputs [2]. But, the minimization is achieved under an additional constraint that the required rate should be provided to each user in each slot irrespective of its channel state. Thus, these schemes can not benefit from users' channel variability over time. Recently, we have shown that the significant power saving can be achieved by exploiting a small delay tolerance of the application [6]. In absence of a specific delay constraint, the proposed scheme is shown to minimize power while guaranteeing the desired throughput and bounded delay for each user. The optimality result has been shown in asymptotic case, i.e., as the number of users go to infinity [6]. Optimality for the finite users case has remained open.

For finite users case, [7], [8], [9] have found back-pressure based scheduling strategies to minimize the energy consumption in the wireless system with ergodic fading while providing the required 
throughputs and bounded delays to the users. These schemes assume that the coding strategy is predefined and for the given coding strategy determine the rate to be provided in each slot by solving an optimization problem. The optimization problem may be non-linear depending on coding/signaling strategy used, and hence may become computationally expensive in practice.

Here, we consider the finite users case, and propose a computationally simple power optimal scheme that provides the required throughputs and bounded delays to the users. The optimization is over the joint space of coding and rate allocations. Specifically, the proposed optimal policy is back-pressure based policy like that in [7], [8], [9], and employees super-position encoding and successive decoding in each slot. The proposed policy arbitrates scheduling based only on the users' current backlogs and the channel states. In spite of using this limited information, it is shown to be optimal even in the class of offline policies that take into account the channel states and arrivals in past, present and even the future slots. One of the main challenges in execution of the proposed policy is that the optimal rate allocation can only be obtained by solving a convex optimization in every slot. But, we obtain a computationally simple algorithm that exploits the problem structure and solves the optimization. All the results extend in straightforward fashion to the broadcast case because of uplink-downlink duality [10].

The paper is arranged as follows. In Section II we present our system model. In Section III, we present some known results that we use. In Section IV] we propose our optimal policy and prove its optimality. In Section $\nabla$, we conclude.

\section{SySTEM MOdel}

We consider a multi-access channel with $N$ users. Time is slotted. For each user $k$, let $\left\{A_{k}(t)\right\}_{t \geq 1}$ denote the random process of arrivals, i.e., $A_{k}(t)$ denote the arrivals for $k$ in slot $t$. We assume that $\vec{A}(t)=\left[\begin{array}{llll}A_{1}(t) & \cdots & A_{N}(t)\end{array}\right]$ are the independent and identically distributed (i.i.d.) random vectors across the slots. Moreover, let $a_{k}=\mathbb{E}\left[A_{k}(t)\right]$. Alternatively, $a_{k}$ denotes the throughput requirement of user $k$. We assume that $a_{k}<\infty$ for every $k$. The arrivals for each user $k$ are queued in the infinite capacity buffer. We denote by $\vec{Q}(t)=\left[Q_{1}(t) \cdots Q_{N}(t)\right]$, where $Q_{k}(t)$ is the backlog or queue length for user $k$ in slot $t$, i.e., $Q_{k}(t)$ is the difference between the total arrivals minus the total departures until time $t$.

Now, we describe our channel model. We assume multi-band system. Specifically, we assume that there are $M$ non-interfering bands available for communication. Let $\vec{d}_{k}(t)=\left[\begin{array}{llll}d_{k, 1}(t) & \cdots & d_{k, M}(t)\end{array}\right]$ denote the vector of channel gains for user $k$ in slot $t$ on each of the bands. Thus, if $E_{k, m}(t)$ denotes the transmit energy per symbol for user $k$ on sub-band $m$ in slot $t$, then the received energy on the sub-band is given by $d_{k, m}(t) E_{k, m}(t)$. We assume that $\left\{\vec{d}_{k}(t): k=1, \ldots, n\right\}_{t \geq 1}$ is a positive 
recurrent finite state Markov process. Note that this assumption is not restrictive as correlated Rician and Rayleigh fading channels can be modeled reasonably well using a finite state Markov process [11], [12]. Let $N_{0}$ denote the noise power spectral efficiency.

Let $R_{k, m}(t)$ denote the service rate for user $k$ on sub-band $m$ in slot $t$. Then, for every $k$, the queue length dynamics is characterized by

$$
Q_{k}(t+1)=\max \left\{Q_{k}(t)+A_{k}(t)-\sum_{m=1}^{M} R_{k, m}(t), 0\right\} .
$$

Clearly, $R_{k, m}(t)$ depends on the channel gains, transmit energies and the coding strategy used. We consider the space of coding strategies such that the rates achieved on sub-band $m$ is independent of the rates on the other sub-bands. Alternatively, communications on various sub-bands are independent. Note that the communication on the same sub-band for various users may not be independent.

Definition 1 (Scheduling Strategy): A scheduling policy $\Delta$ arbitrates the rate allocation $R_{k, m}(t)$ and coding strategy for every user $k$ and sub-band $m$ in every slot $t$.

This class includes offline policies that decide their rate allocation and coding based on the knowledge of arrivals and channel states in each past, present and even future slots.

We assume that $\vec{Q}(t)$ and $\vec{d}_{k}(t)$ for every $k$ is known and a scheduling policy can utilize this knowledge in its decision process. In case of a possible ambiguity, we use superscript $\Delta$ to indicate the dependence of various terms on $\Delta$, e.g., $R_{k, m}^{\Delta}(t)$ and $E_{k, m}^{\Delta}(t)$ will denote the rate and transmit energy respectively for user $k$ in sub-band $m$ in slot $t$ under $\Delta$.

Definition 2 (Stability): The multi-access system is said to be stable if the mean queue length in every slot $t$ for every user $k$ is upper bounded by a number that is independent on $t$, i.e., $\sup _{t \geq 1}\left\{\mathbb{E}\left[Q_{k}(t)\right]\right\}<\infty$ for every $k$. A scheduling policy that stabilizes the system is called stable scheduling policy.

Note that every stable scheduling policy guarantees the required throughput $a_{k}$ to every user $k$, and in addition, guarantees bounded delay for the arrivals.

Definition 3 (Power Efficiency): The power efficiency of scheduling policy $\Delta$ is defined as

$$
P^{\Delta}=\limsup _{T \rightarrow \infty} \frac{1}{T} \sum_{t=1}^{T} \sum_{k=1}^{N} \sum_{m=1}^{M} E_{k, m}^{\Delta}(t) .
$$

Definition 4 (Optimality): A stable policy $\Delta$ is said to be optimal if with probability (w.p.) 1 it attains the smallest power efficiency among all the stable policies.

Let $P_{\min }(\mathcal{C})$ be the infimum of the power efficiencies of all the stable policies in a class $\mathcal{C}$ of scheduling policies. If $\mathcal{C}$ does not contain any stable policy, then $P_{\min }(\mathcal{C})$ is defined to be $\infty$. Furthermore, let $P_{\min }$ denote the optimal power efficiency, i.e., $P_{\min }=P_{\min }(\mathcal{C})$ where $\mathcal{C}$ is the set of all policies. 
Definition 5 ( $\epsilon$-optimality): A scheduling policy $\Delta$ is said to be $\epsilon$-optimal in class $\mathcal{C}$ of scheduling policies if it is stable and $P^{\Delta} \leq P_{\min }(\mathcal{C})+\epsilon$ w.p. 1 . Moreover, $\Delta$ is said to be $\epsilon$-optimal if it is stable and $P^{\Delta} \leq P_{\min }+\epsilon$ w.p. 1 .

\section{BACKGROUND}

We present the following known results for the sake of completeness. To be consistent, we state these results in the notation introduced here.

Fix a sequence of coding strategies in every slot and let $\mathcal{C}$ denote the class of scheduling policies that use this fixed sequence of the coding strategies. Also, let $\Delta_{1}(V) \in \mathcal{C}$ denote a parametrized scheduling policy that assigns the rates by solving the optimization problem

Minimize: $\sum_{m=1}^{M}\left[\sum_{k=1}^{N} V E_{k, m}(t)-\sum_{k=1}^{N} Q_{k}(t) R_{k, m}(t)\right]$

Subject to: $R_{k, m}(t) \geq 0$ for every $k$ and $m$,

where $V$ is a fixed constant. Then, the following are the performance guarantees for $\Delta_{1}(V)$.

Theorem 1 (Result from [7], [8], [9]): For every $\epsilon>0$, there exists $\widehat{V}>0$ such that for every $V>\widehat{V}, \Delta_{1}(V)$ is $\epsilon$-optimal in $\mathcal{C}$.

We present the intuition for the result. Consider a case when $Q_{k}(t)$ is much smaller than $V$. Broadly, it implies that the user was receiving the desired rate in the past. Thus, $\Delta_{1}(V)$ provides positive rate to the user only if the corresponding energy cost is much smaller, i.e., when the user's channel gain is large. On the contrary, if $Q_{k}(t)$ is much larger than $V$, then it implies that the user was not receiving the desired rate and also that the user's average channel gain is small. Thus, $\Delta_{1}(V)$ provides positive rate to the user even when the user has, potentially, small channel gain in order to preserve stability. Alternatively, the current queue length represents the history of the rate provided to the user and its channel quality. Thus, $\Delta_{1}(V)$ estimates users' desired throughput and channel quality using the current queue length, and then invests just enough power to maintain stability.

Given coding strategies, Theorem 1 provides a way to obtain $\epsilon$-optimal policies. Thus, it remains to determine how optimal coding strategy can be obtained in every slot. The following theorem provides useful guidelines in this direction.

Theorem 2 (Results in [2]): For a given rate assignment $R_{1}, \ldots, R_{N}$ and channel states $d_{1}, \ldots, d_{N}$ the total sum energy $\sum_{k=1}^{N} E_{k}$ required to realize the rates is minimized by super-position coding and successive decoding. Moreover, for optimal signaling, the successive decoding order depends only on channel gains, but not on the rate assignment.

Let $\vec{\pi}$ denote the permutation that sorts the gains in the increasing order, i.e., $d_{\pi_{1}} \leq d_{\pi_{2}} \leq \cdots \leq$ $d_{\pi_{N}}$. Then, the required transmit energy per symbol for user $\pi_{k}$ is given by

$$
E_{\pi_{k}}=\frac{N_{0}}{d_{\pi_{k}}}\left[e^{R_{\pi_{k}}}-1\right] e^{\sum_{i<k} R_{\pi_{i}}}
$$




\section{IV. $\epsilon$-OPTIMAL SCHEDULING POLICY}

Let us define the following function for a fixed constant $V$.

$$
F\left(\vec{R}_{m}(t)\right) \stackrel{\text { def }}{=} \sum_{k=1}^{N} \frac{V N_{0}}{d_{\pi_{k}^{m}, m}(t)}\left[e^{R_{\pi_{k}^{m}, m}(t)}-1\right] e^{\sum_{i<k} R_{\pi_{i}^{m}, m}(t)}-\sum_{k=1}^{N} Q_{\pi_{k}^{m}}(t) R_{\pi_{k}^{m}, m}(t),
$$

where $\vec{\pi}^{m}$ is a permutation that sorts the gains on sub-band $m$ in the increasing order. Now, let us consider a parametrized scheduling policy $\Delta^{*}(V)$ that assigns in every slot the rates $R_{k, m}(t)$ that solve

Optimization (O1) - Minimize: $\sum_{m=1}^{M} F\left(\vec{R}_{m}(t)\right)$

Subject to: $R_{k, m}(t) \geq 0$ for every $k$ and $m$,

and then achieves the rates using super-position coding and successive decoding on each sub-band separately. Clearly, $R_{k, m}^{\Delta^{*}(V)}(t)=0$ for every $m$, if $Q_{k}(t)=0$. We show the following optimality result for $\Delta^{*}(V)$.

Theorem 3: For every $\epsilon>0$, there exists $\widehat{V}>0$ such that for every $V>\widehat{V}, \Delta^{*}(V)$ is $\epsilon$-optimal.

Proof: Let $\mathcal{C}^{*}$ denote the class of scheduling policies that use super-position coding and successive decoding in every slot. Then, we show that $P_{\min }=P_{\min }\left(\mathcal{C}^{*}\right)$.

Let $\Delta_{1}$ denote any stable policy. Now, we construct $\Delta_{2} \in \mathcal{C}^{*}$ as follows. For every $k, m$ and $t$ choose $R_{k, m}^{\Delta_{2}}(t)=R_{k, m}^{\Delta_{1}}(t)$. Clearly, $\Delta_{2}$ is also stable. Moreover, by Theorem 2, for every $t$ $\sum_{m=1}^{M} \sum_{k=1}^{N} E_{k, m}^{\Delta_{2}}(t) \leq \sum_{m=1}^{M} \sum_{k=1}^{N} E_{k, m}^{\Delta_{1}}(t)$. Thus, by Definition 3, $P^{\Delta_{1}} \geq P^{\Delta_{2}}$. Since, $\Delta_{1}$ is an arbitrary stable scheduling policy, we conclude that $P_{\min }=P_{\min }\left(\mathcal{C}^{*}\right)$.

Now, the result follows from Theorem 1 and (1).

Note that Theorem 3 provides a way to minimize power while stabilizing the system. The minimization is over the space of all coding and rate assignment strategies. The policy $\Delta^{*}(V)$ achieves the optimality by taking into account only the current system state, and does not require the knowledge of statistics of the arrival and channel processes a priori. Moreover, optimality holds among the class of off-line scheduling policies. In spite of these desirable properties, $\Delta^{*}(V)$ has one major limitation which is that it needs to solve a non-linear optimization (O1) in every slot to obtain the optimal rate assignment. Solving (O1) may be computationally expensive, and thereby limit the practicality of $\Delta^{*}(V)$. In the following discussion, we focus on (O1) and derive certain properties of the optimal solution and using these propose an algorithm that obtains optimal rate allocation with polynomial complexity.

Since the communication on each of the sub-bands is independent, to solve (O1), it suffices to solve separately for every $m$

Optimization (O2) - Minimize: $F\left(\vec{R}_{m}(t)\right)$

Subject to: $R_{k, m}(t) \geq 0$ for every $k$. 
Moreover, since the nature of optimization (objective function and constraints) is identical for every $m$, an algorithm to solve (O2) for a given $m$ can be utilized for all $m$ 's. So, we fix $m$ and $t$ and focus on (O2).

In the following discussion, for notational brevity, we omit $m$ and $t$. Also, without loss of generality, let $\pi_{k}^{m}=k$. With this simplified notation (O2) becomes

Minimize: $F(\vec{R})=\sum_{k=1}^{N} \frac{V N_{0}}{d_{k}}\left[e^{R_{k}}-1\right] e^{\sum_{i<k} R_{i}}-\sum_{k=1}^{N} Q_{k} R_{k}$

Subject to: $R_{k} \geq 0$ for every $k$.

Note that (O2) is strictly convex (see Appendix \). This can be verified by checking that the Hessian is positive definite in the positive half plane [13]. For convex optimization, polynomial complexity algorithms using the interior point method have been proposed [14]. These algorithms obtain a solution within $\delta>0$ neighborhood of the optimal value. The computational complexity of these algorithms is $O\left(N^{3}\right)$ per accuracy digit [14]. We, however, propose the $O\left(N^{2}\right)$ complexity algorithm that computes the exact optimal solution.

We start by looking at the Lagrange relaxation of (O2).

Minimize: $F(\vec{R}, \vec{\lambda})=\sum_{k=1}^{N} \frac{V N_{0}}{d_{k}}\left[e^{R_{k}}-1\right] e^{\sum_{i<k} R_{i}}-\sum_{k=1}^{N}\left(Q_{k}+\lambda_{k}\right) R_{k}$, where $\vec{\lambda}=\left\{\lambda_{1}, \ldots, \lambda_{N}\right\}$ are Lagrange multipliers. Now, for every $k$

$$
\frac{\partial F(\vec{R}, \vec{\lambda})}{\partial R_{k}}=\sum_{i=k+1}^{N} \frac{V N_{0}}{d_{i}}\left(e^{R_{i}}-1\right) e^{\sum_{u=1}^{i-1} R_{u}}+\frac{V N_{0}}{d_{k}} e^{\sum_{i=1}^{k} R_{i}}-\left(Q_{k}+\lambda_{k}\right) .
$$

Lemma 1: The following relations satisfy $\frac{\partial F(\vec{R}, \vec{\lambda})}{\partial R_{k}}=0$ for every $k$.

$$
\begin{aligned}
& R_{k}=\log \left(\frac{\left[\left(Q_{k}+\lambda_{k}\right)-\left(Q_{k+1}+\lambda_{k+1}\right)\right]\left[\frac{1}{d_{k-1}}-\frac{1}{d_{k}}\right]}{\left[\left(Q_{k-1}+\lambda_{k-1}\right)-\left(Q_{k}+\lambda_{k}\right)\right]\left[\frac{1}{d_{k}}-\frac{1}{d_{k+1}}\right]}\right) \text { for } k>1 \\
& R_{1}=\log \left(\frac{\left(Q_{1}+\lambda_{1}\right)-\left(Q_{2}+\lambda_{2}\right)}{V N_{0}\left[\frac{1}{d_{1}}-\frac{1}{d_{2}}\right]}\right),
\end{aligned}
$$

by defining $d_{N+1}=\infty$ and $Q_{N+1}=\lambda_{N+1}=0$.

Proof: We show the required by proving that for every $k, \frac{\partial F(\vec{R}, \vec{\lambda})}{\partial R_{k}}=0$ implies

$$
e^{\sum_{u \leq k} R_{u}}=\frac{\left(Q_{k}+\lambda_{k}\right)-\left(Q_{k+1}+\lambda_{k+1}\right)}{V N_{0}\left[\frac{1}{d_{k}}-\frac{1}{d_{k+1}}\right]} .
$$

We prove the above using induction on $k$.

As a base case we show (5) for $k=N$. Note that substituting $k=N$ in (2) and equating it to 0 , we obtain (5). Thus, (5) holds for $k=N$. Now, for induction, we assume that (5) holds for every $k \geq s+1$ and verify it for $k=s$. 
Consider the second term in (2) with $k=s$.

$$
\begin{aligned}
& \sum_{i=s+1}^{N} \frac{V N_{0}}{d_{i}}\left(e^{R_{i}}-1\right) e^{\sum_{u=1}^{i-1} R_{u}} \\
& =\sum_{i=s+1}^{N} \frac{V N_{0}}{d_{i}} e^{\sum_{u=1}^{i} R_{u}}-\sum_{i=s+1}^{N} \frac{V N_{0}}{d_{i}} e^{\sum_{u=1}^{i-1} R_{u}} \\
& =\sum_{i=s+1}^{N-1} V N_{0}\left[\frac{1}{d_{i}}-\frac{1}{d_{i+1}}\right] e^{\sum_{u=1}^{i} R_{u}}+\frac{V N_{0}}{d_{N}} e^{\sum_{u=1}^{N} R_{u}}-\frac{V N_{0}}{d_{s+1}} e^{\sum_{u=1}^{s} R_{u}} \\
& =\left(Q_{s+1}+\lambda_{s+1}\right)-\frac{V N_{0}}{d_{s+1}} e^{\sum_{u=1}^{s} R_{u}} .
\end{aligned}
$$

Last equality follows from (5) and the induction hypothesis. Now, substituting the above in (2), we obtain the desired.

Finally, (3) follows by observing $R_{k}=\log \left(\frac{e^{\sum_{u=1}^{k} R_{u}}}{e^{\sum_{u=1}^{k-1} R_{u}}}\right)$ and (4) is obtained directly from (5) with $k=1$.

Definition 6 (From [15], pp. 328): The vectors $\vec{R}^{\prime}$ and $\vec{\lambda}^{\prime}$ are said to satisfy Karush-Kuhn-Tucker (KKT) conditions if they satisfy the following relations.

$$
\begin{aligned}
\left.\frac{\partial F(\vec{R}, \vec{\lambda})}{\partial R_{k}}\right|_{\vec{R}=\vec{R}^{\prime}} & =0 \text { for every } k \\
\vec{R}^{\prime} & \geq \overrightarrow{0} \\
\vec{\lambda}^{\prime} & \geq \overrightarrow{0} \\
R_{k}^{\prime} \lambda_{k}^{\prime} & =0 \text { for every } k .
\end{aligned}
$$

Since (O2) is strictly convex in the feasible region, we conclude the following [15].

1) The optimal solution is unique.

2) The rate allocation $\vec{R}^{\prime}$ is optimal iff there exists $\vec{\lambda}^{\prime}$ such that $\vec{R}^{\prime}$ and $\vec{\lambda}^{\prime}$ satisfy the KKT conditions. Also, such $\vec{\lambda}^{\prime}$ is unique since linear independence constraint qualification holds.

In Figure 1, we propose a general procedure for obtaining a rate allocation $\vec{R}$ and Lagrange multipliers $\vec{\lambda}$ that satisfy the KKT conditions for any given $\vec{Q}$ and $\vec{d}$. We first intuitively describe the proposed algorithm and subsequently prove that the algorithm optimally solves (O2).

The main procedure Computation_of_Optimal_Rates takes current queue length vector $\vec{Q}$ and the channel gains $\vec{d}$ as input and outputs the optimal rate allocation $\vec{R}$. In this procedure, we define two sets $\mathcal{A}$ and $\mathcal{E}$ that partition the set of all users. The set $\mathcal{A}(\mathcal{E}$, resp.) denotes the set of active (inactive, resp.) users. A user $k$ is said to be active if $R_{k}>0$, i.e., it is served at positive rate; $k$ is inactive otherwise. Initially, all the users are assumed to be active (Line 1). Next, the algorithm iterates and in each iteration determines an inactive user using (10) and (11) (Line 2). Once the inactive user is determined the sets $\mathcal{A}$ and $\mathcal{E}$ are updated (Lines 3 and 4 ), and subsequently the Lagrange multipliers 
Computation_of_Optimal_Rates $(\vec{Q}, \vec{d})$

begin

1: Initialize $\mathcal{A} \leftarrow\{1, \ldots, N\}, \mathcal{E} \leftarrow \phi$ and $\vec{\lambda} \leftarrow \overrightarrow{0}$

2: while There exists $k \in \mathcal{A}$ such that

$$
\begin{aligned}
Q_{k} & <\frac{\left(Q_{k-1}+\lambda_{k-1}\right)\left[\frac{1}{d_{k}}-\frac{1}{d_{k+1}}\right]+\left(Q_{k+1}+\lambda_{k+1}\right)\left[\frac{1}{d_{k-1}}-\frac{1}{d_{k}}\right]}{\left[\frac{1}{d_{k-1}}-\frac{1}{d_{k+1}}\right]} \text { for } k>1 \\
Q_{1} & <V N_{0}\left[\frac{1}{d_{1}}-\frac{1}{d_{2}}\right]+\left(Q_{2}+\lambda_{2}\right)
\end{aligned}
$$

do

$$
\begin{aligned}
\text { 3: } & \mathcal{E} \leftarrow \mathcal{E} \cup\{k\} \\
\text { 4: } & \mathcal{A} \leftarrow \mathcal{A}-\{k\} \\
\text { 5: } & \text { Update_Lagrange_Multipliers }(\mathcal{A}, \mathcal{E}) \\
& \{/ * \text { Optimal Rate computation } * /\} \\
\text { 6: } & R_{k} \leftarrow 0 \text { for every } k \in \mathcal{E} \\
\text { 7: } & \text { Compute } R_{k} \text { for every } k \in \mathcal{A} \text { using }
\end{aligned}
$$

end

Update_Lagrange $\_$Multipliers $(\mathcal{A}, \mathcal{E})$

begin

1: $\lambda_{k} \leftarrow 0$ for every $k \in \mathcal{E}$

2: if $\{1, \ldots, u-1\} \subseteq \mathcal{E}$ and $u \in \mathcal{A}$ then

3: $\quad$ for every $m \in\{1, \ldots, u-1\}$

$$
\lambda_{m} \leftarrow V N_{0}\left[\frac{1}{d_{m}}-\frac{1}{d_{u}}\right]+\left(Q_{u}-Q_{m}\right) .
$$

4: if $\{v+1, \ldots, u-1\} \subseteq \mathcal{A}$ and $\{v, u\} \subseteq \mathcal{E}$ then

5: $\quad$ for every $m \in\{v+1, \ldots, u-1\}$

$$
\lambda_{m} \leftarrow \frac{Q_{v}\left[\frac{1}{d_{m}}-\frac{1}{d_{u}}\right]+Q_{u}\left[\frac{1}{d_{v}}-\frac{1}{d_{m}}\right]}{\left[\frac{1}{d_{v}}-\frac{1}{d_{u}}\right]}-Q_{m} .
$$

end

Fig. 1. Figure shows the pseudo code of an algorithm that computes the optimal rate allocation in a given slot

are also updated (Line 5). If no user in $\mathcal{A}$ satisfy (10) and (11), then the algorithm terminates after computing the rate allocation using (3) and (4) (Lines 6 and 7). This ensures that (6) is satisfied for all $k \in \mathcal{E}$. Now, we explain why a user satisfying (10) or (11) should be inactive. Note that (10) and (11) are equivalent to $R_{k}<0$ in (3) and (4), respectively. Since the assigned rates can only be non-negative, we put such a user $k$ in $\mathcal{E}$ and update corresponding $\lambda_{k}$ so as to ensure $R_{k}=0$.

Now, we briefly explain how the procedure Update_Lagrange_Multipliers computes Lagrange multipliers in each iteration. Note that for every active user $k, \lambda_{k}$ must be zero in order to satisfy the KKT condition (9). Thus in the first step, the procedure assigns $\lambda_{k}=0$ for every $k \in \mathcal{A}$ (Line 1). 
Next, for every $k \in \mathcal{E}$, it computes $\lambda_{k}$ so that $R_{k}$ in (3) or (4) equals zero (Lines 2 to 5 ). This ensures that (6), (7) and (9) hold for every $k \in \mathcal{A}$. We need to recompute all the Lagrange multipliers in every iteration because the value of $\lambda_{k}$ is a function of $\lambda_{k-1}$ and $\lambda_{k+1}$ as can be seen from (3) and (4).

Even though the algorithm is straightforward, mainly, two questions are unanswered. First, whether $\lambda_{k}$ is non-negative for every $k \in \mathcal{E}$. Second, since the $\lambda_{k}$ 's for many users (not only the recently added user) in $\mathcal{E}$ are updated, how is it ensured that an inactive user does not become active in the subsequent iterations. We formally address these questions and prove the optimality of the proposed algorithm.

For analysis, we introduce the following additional notation. Let $\vec{R}^{*}$ and $\vec{\lambda}^{*}$ denote the rate vector and Lagrange multipliers computed by the algorithm at termination. Also, let $\mathcal{A}^{*}$ and $\mathcal{E}^{*}$ denote the sets $\mathcal{A}$ and $\mathcal{E}$, respectively, when the algorithm terminates. Next, we distinguish between the value of $\vec{\lambda}, \mathcal{A}$ and $\mathcal{E}$ computed by the algorithms in every iteration. Let $\vec{\lambda}^{i}, \mathcal{A}^{i}$ and $\mathcal{E}^{i}$ denote $\vec{\lambda}, \mathcal{A}$ and $\mathcal{E}$, respectively, computed by the algorithm in $i^{\text {th }}$ iteration. Because of the initialization in Line 1 of procedure Computation_of_Optimal_Rates, $\vec{\lambda}^{0}=\overrightarrow{0}, \mathcal{A}^{0}=\{1, \ldots, N\}$ and $\mathcal{E}^{0}=\phi$. Let the algorithm terminate in $I$ iterations. Then, clearly, $I \leq N$ and $\vec{\lambda}^{I}=\vec{\lambda}^{*}, \mathcal{A}^{I}=\mathcal{A}^{*}$ and $\mathcal{E}^{I}=\mathcal{E}^{*}$. Now, we show the following result.

Lemma 2: If $\vec{\lambda}^{*} \geq \overrightarrow{0}$, then $\vec{R}^{*}$ and $\vec{\lambda}^{*}$ satisfy the KKT conditions.

Proof: Note that for every $k \in \mathcal{A}^{*}, R_{k}^{*}$ is computed using (3) and (4). Thus by Lemma 1 , clearly, (6) is satisfied for every $k \in \mathcal{A}^{*}$. Now, we show that (6) also holds for every $k \in \mathcal{E}^{*}$. Note that $R_{k}^{*}=0$ for every $k \in \mathcal{E}^{*}$. Thus, it suffices to show that when the chosen $\vec{\lambda}^{*}$ is substituted in (3) and (4) yields $R_{k}^{*}=0$ for every $k \in \mathcal{E}^{*}$. The required can be easily verified using elementary algebra. Thus (6) holds for every $k$.

Now, we show that $\vec{\lambda}^{*}$ satisfy (7). Since, $R_{k}^{*}=0$ for every $k \in \mathcal{E}^{*}$, (7) clearly holds for every $k \in \mathcal{E}^{*}$. Now, we show (7) for every $k \in \mathcal{A}^{*}$. We show the required using contradiction. Let there be $k \in \mathcal{A}^{*}$ such that $R_{k}^{*}<0$. But then from (3) and (4) it implies that

$$
\begin{aligned}
Q_{k}<\frac{\left(Q_{k-1}+\lambda_{k-1}^{*}\right)\left[\frac{1}{d_{k}}-\frac{1}{d_{k+1}}\right]+\left(Q_{k+1}+\lambda_{k+1}^{*}\right)\left[\frac{1}{d_{k-1}}-\frac{1}{d_{k}}\right]}{\left[\frac{1}{d_{k-1}}-\frac{1}{d_{k+1}}\right]} \text { if } k>1 \\
Q_{k}<V N_{0}\left[\frac{1}{d_{k}}-\frac{1}{d_{k+1}}\right]+\left(Q_{k+1}+\lambda_{k+1}^{*}\right) \quad \text { if } k=1 .
\end{aligned}
$$

Now, from (10) and (11), we conclude that the algorithm will not terminate, but instead add $k$ to $\mathcal{E}$ and continue. Thus, no such index exists. So, $\vec{\lambda}^{*}$ and $\vec{R}^{*}$ satisfy (7).

The vectors $\vec{\lambda}^{*}$ and $\vec{R}^{*}$ satisfy (8) because of the supposition in the lemma. Moreover, the vectors satisfy (9) because $R_{k}^{*}=0$ for every $k \in \mathcal{E}^{*}$, while $\lambda_{k}^{*}=0$ for every $k \in \mathcal{A}^{*}$. 
In the following theorem, we show that $\vec{\lambda}^{*}$ is non-negative.

Theorem 4: For every $i<I, \vec{\lambda}^{i} \leq \vec{\lambda}^{i+1}$.

Note that since $\vec{\lambda}^{0}=\overrightarrow{0}$, Theorem 4 implies that $\vec{\lambda}^{*} \geq \overrightarrow{0}$. We prove the above theorem by showing the required in each of the cases that may be arise in the execution of the algorithm. The proofs use elementary algebra. For better readability, proofs for all the cases are given in Appendix 【

Finally, we prove the optimality of the proposed algorithm.

Theorem 5: The rate allocation $\vec{R}^{*}$ is the unique optimal solution of (O2).

Proof: The result follows immediately from the strict convexity of (O2), Lemma 2 and Theorem 4

\section{CONCLUSION}

We have considered a multi-access channel with $N$-users. We have proposed a parametrized scheduling policy $\Delta^{*}(V)$ which is $\epsilon$-optimal for every $\epsilon>0$ for appropriate choice of the parameter $V$ even among the offline strategies in spite of considering only the current queue lengths and channel gains in its decision process. Moreover, the optimization is over the joint space of coding and rate allocation strategies. The policy $\Delta^{*}(V)$ needs to solve a convex optimization in every slot to obtain the optimal rate allocation. We have proposed a $O\left(N^{2}\right)$ algorithm that accurately solves the optimization. All the results extend in straightforward fashion to broadcast case because of uplink-downlink duality.

\section{REFERENCES}

[1] D. Tse and S. Hanly, "Multi-access fading channels-part i: Polymatroid structure, optimal resource allocation and throughput capacities," IEEE Transactions on Information Theory, vol. 44, no. 7, pp. 2796-2815, Nov. 1998.

[2] S. Hanly and D. Tse, "Multi-access fading channels-part ii: Delay-limited capacities," IEEE Transactions on Information Theory, vol. 44, no. 7, pp. 2816-2831, Nov. 1998.

[3] P. Bender, P. Black, M. Grob, R. Padovani, N. Sindhushayana, and A. Viterbi, "Cdma/hdr: A bandwidth-efficient high-speed wireless data service for nomadic users," IEEE Commun. Mag., vol. 38, no. 7, pp. 70-77, Jul. 2000.

[4] D. Tse, "Multiuser diversity through proportional fair scheduling," in Communication theory workshop, May 2001.

[5] P. Viswanath, D. Tse, and R. Laroia, "Opportunistic beamforming using dumb antennas," IEEE Transactions on Information Theory, vol. 48, no. 6, Jun. 2002.

[6] P. Chaporkar, K. Kansanen, and R. Müller, "Channel and multiuser diversities in wireless systems: Delayenergy tradeoff," Norwegian University of Science and Technology, Trondheim, Norway, Tech. Rep., Dec 2006, http://www.iet.ntnu.no/ ralf/Publications.html

[7] P. Chaporkar and S. Sarkar, "Stable scheduling policies for maximizing throughput in generalized constrained queueing," in IEEE INFOCOM'06, Barcelona, Spain, Apr. 2006.

[8] M. Neely, "Energy optimal control for time varying wireless networks," in IEEE INFOCOM'05, Mar. 2005.

[9] A. Stolyar, "Maximizing queueing network utility subject to stability: Greedy primal-dual algorithm," Queueing Systems, vol. 50, no. 4, pp. 401-457, Aug. 2005. 
[10] N. Jindal, S. Vishwanath, and A. Goldsmith, "On duality of gaussian multiple-access and broadcast channels," IEEE Transactions on Information Theory, vol. 50, no. 5, pp. 768-783, May 2004.

[11] Q. Zhang and S. Kassam, "Finite state markov model for rayleigh fading channel," IEEE Transactions on communications, vol. 47, no. 11, pp. 1688-1692, Nov 1999.

[12] C. Pimentel, T. Falk, and L. Lisboa, "Finite-state markov modeling of correlated rician-fading channels," IEEE transactions on vehicular technology, vol. 53, no. 5, pp. 1491-1501, May 2004.

[13] S. Boyd and L. Vandenberghe, Convex Optimization. Cambridge University Press, Mar 2004.

[14] Y. Nesterov and A. Nemirovskii, Interior-Point Polynomial Algorithms in Convex Programming, ser. Studies in Applied and Numerical Mathematics. Society for Industrial \& Applied Mathematics, Jul 1995.

[15] J. Nocedal and S. J. Wright, Numerical optimization. New York: Springer-Verlag, 1999.

\section{APPENDIX I}

\section{Convexity of Optimization (O2)}

The second partial derivative of $F(\vec{R})$ is as follows.

$$
\frac{\partial^{2} F(\vec{R})}{\partial R_{k} \partial R_{j}}=\left\{\begin{array}{c}
\sum_{i=k+1}^{N} \frac{V N_{0}}{d_{i}}\left(e^{R_{i}}-1\right) e^{\sum_{u=1}^{i-1} R_{u}}+\frac{V N_{0}}{d_{k}} e^{\sum_{i=1}^{k} R_{i}}: \quad j \leq k \\
\frac{V N_{0}}{d_{j}} e^{\sum_{u=1}^{j} R_{u}}+\sum_{i=j+1}^{N} \frac{V N_{0}}{d_{i}}\left(e^{R_{i}}-1\right) e^{\sum_{u=1}^{i-1} R_{u}}: \quad j>k .
\end{array}\right.
$$

Note that for every $\vec{R} \in[0, \infty)^{N}, \frac{\partial^{2} F(\vec{R})}{\partial R_{k} \partial R_{j}}>0$ for any $k$ and $j$. This shows that the Hessian of $F(\vec{R})$ is positive definite. Also, it is clear that the feasible region $[0, \infty)^{N}$ is a convex set. Thus, (O2) is an instance of convex optimization.

\section{APPENDIX II}

\section{SUPPORTING LEMMAS FOR PROVING TheOREM 4}

Lemma 3: Let index $k$ be added to the set $\mathcal{E}^{i-1}$ in the $i^{\text {th }}$ iteration. Then for all users $u$ such that there exists $v \in \mathcal{A}^{i}$ between $k$ and $u, \lambda_{u}^{i}=\lambda_{u}^{i-1}$.

Proof: The proof follows immediately from the procedure Update_Lagrange_Multipliers in Figure 1.

Lemma 4: Let index $k$ be added to the set $\mathcal{E}^{i-1}$ in the $i^{\text {th }}$ iteration. Also, let $\{k-1, k+1\} \in \mathcal{A}^{i-1}$. Then, $\lambda_{n}^{i}-\lambda_{n}^{i-1} \geq 0$ for every $n$.

Proof: Since index $k$ is added to $\mathcal{E}^{i-1}$ in the $i^{\text {th }}$ iteration, we know the following. First, $\lambda_{k}^{i-1}=0$. Second, from (10)

$$
Q_{k}<\frac{\left.\left(Q_{k-1}+\lambda_{k-1}^{i-1}\right)\left[\frac{1}{d_{k}}-\frac{1}{d_{k+1}}\right]+\left(Q_{k+1}+\lambda_{k+1}^{i-1}\right)\right)\left[\frac{1}{d_{k-1}}-\frac{1}{d_{k}}\right]}{\left[\frac{1}{d_{k-1}}-\frac{1}{d_{k+1}}\right]} .
$$

Note that $\lambda_{k-1}^{i-1}=\lambda_{k+1}^{i-1}=0$. Thus the result follows from (13), (14) and Lemma 3 ,

Lemma 5: Let index 1 be added to the set $\mathcal{E}^{i-1}$ in the $i^{\text {th }}$ iteration. Also, let $\{2, \ldots, m-1\} \subseteq \mathcal{E}^{i-1}$ and $m \notin \mathcal{E}^{i-1}$. Then, $\lambda_{n}^{i}-\lambda_{n}^{i-1} \geq 0$ for every $n$. 
Proof: Since index 1 is added to $\mathcal{E}^{i-1}$ in the $i^{\text {th }}$ iteration, we know the following. First, $\lambda_{1}^{i-1}=0$. Second, from (11)

$$
Q_{1}<V N_{0}\left[\frac{1}{d_{1}}-\frac{1}{d_{2}}\right]+\left(Q_{2}+\lambda_{2}^{i-1}\right)
$$

Moreover, since $\{2, \ldots, m-1\} \subseteq \mathcal{E}^{i-1}$ and $m \notin \mathcal{E}^{i-1}$, we also know that for every $n \in\{2, \ldots, m-$ $1\}$,

$$
\lambda_{n}^{i-1}=\frac{Q_{1}\left[\frac{1}{d_{n}}-\frac{1}{d_{m}}\right]+Q_{m}\left[\frac{1}{d_{1}}-\frac{1}{d_{n}}\right]}{\left[\frac{1}{d_{1}}-\frac{1}{d_{m}}\right]}-Q_{n} .
$$

Now, substituting $\lambda_{2}^{i-1}$ from (16) in (15), we obtain

$$
\begin{aligned}
Q_{1} & <V N_{0}\left[\frac{1}{d_{1}}-\frac{1}{d_{2}}\right]+\frac{Q_{1}\left[\frac{1}{d_{2}}-\frac{1}{d_{m}}\right]+Q_{m}\left[\frac{1}{d_{1}}-\frac{1}{d_{2}}\right]}{\left[\frac{1}{d_{1}}-\frac{1}{d_{m}}\right]} \\
\Longrightarrow Q_{1} & <V N_{0}\left[\frac{1}{d_{1}}-\frac{1}{d_{m}}\right]+Q_{m} .
\end{aligned}
$$

Now, note that from (12), for every $n=1, \ldots, m-1$

$$
\lambda_{n}^{i}=V N_{0}\left[\frac{1}{d_{n}}-\frac{1}{d_{m}}\right]+\left(Q_{m}-Q_{n}\right) .
$$

From (17), clearly, $\lambda_{1}^{i}>0$. Now, from (16) and (18), it follows that for every $n=2, \ldots, m-1$

$$
\begin{aligned}
\lambda_{n}^{i}-\lambda_{n}^{i-1} & =V N_{0}\left[\frac{1}{d_{n}}-\frac{1}{d_{m}}\right]+\left(Q_{m}-Q_{n}\right)-\frac{Q_{1}\left[\frac{1}{d_{n}}-\frac{1}{d_{m}}\right]+Q_{m}\left[\frac{1}{d_{1}}-\frac{1}{d_{n}}\right]}{\left[\frac{1}{d_{1}}-\frac{1}{d_{m}}\right]}+Q_{n} \\
& =\frac{\left[\frac{1}{d_{n}}-\frac{1}{d_{m}}\right]}{\left[\frac{1}{d_{1}}-\frac{1}{d_{m}}\right]} \lambda_{1}^{i} \geq 0 .
\end{aligned}
$$

The last inequality follows from the fact that $d_{k} \leq d_{k+1}$ for every $k$ and $\lambda_{1}^{i}>0$. Furthermore, by Lemma 3 , for $n \notin\{1, \ldots, m-1\}, \lambda_{n}^{i-1}=\lambda_{n}^{i}$. Thus, the result follows.

Lemma 6: Let index $k>1$ be added to the set $\mathcal{E}^{i-1}$ in the $i^{\text {th }}$ iteration. Also, let $\{v+1, \ldots, k-1\} \subseteq$ $\mathcal{E}^{i-1}$ and $\{v, k+1\} \subseteq \mathcal{A}^{i}$. Then, $\lambda_{n}^{i}-\lambda_{n}^{i-1} \geq 0$ for every $n$.

Proof: Since index $k$ is added to $\mathcal{E}^{i-1}$ in the $i^{\text {th }}$ iteration, we know the following. First, $\lambda_{k}^{i-1}=0$. Second, from (10)

$$
Q_{k}<\frac{\left.\left(Q_{k-1}+\lambda_{k-1}^{i-1}\right)\left[\frac{1}{d_{k}}-\frac{1}{d_{k+1}}\right]+\left(Q_{k+1}+\lambda_{k+1}^{i-1}\right)\right)\left[\frac{1}{d_{k-1}}-\frac{1}{d_{k}}\right]}{\left[\frac{1}{d_{k-1}}-\frac{1}{d_{k+1}}\right]} .
$$

Moreover, since $\{v+1, \ldots, k-1\} \subseteq \mathcal{E}^{i-1}$ and $\{v, k, k+1\} \subseteq \mathcal{A}^{i-1}$, we also know that for every $n \in\{v+1, \ldots, k-1\}$,

$$
\lambda_{n}^{i-1}=\frac{Q_{v}\left[\frac{1}{d_{n}}-\frac{1}{d_{k}}\right]+Q_{k}\left[\frac{1}{d_{v}}-\frac{1}{d_{n}}\right]}{\left[\frac{1}{d_{v}}-\frac{1}{d_{k}}\right]}-Q_{n} .
$$


Now, substituting $\lambda_{k+1}^{i-1}=0$ and $\lambda_{k-1}^{i-1}$ from (20) in (19), we obtain

$$
\begin{aligned}
& Q_{k}< \frac{\left.\frac{Q_{v}\left[\frac{1}{d_{k-1}}-\frac{1}{d_{k}}\right]+Q_{k}\left[\frac{1}{d_{v}}-\frac{1}{d_{k-1}}\right]}{\left[\frac{1}{d_{v}}-\frac{1}{d_{k}}\right]}\left[\frac{1}{d_{k}}-\frac{1}{d_{k+1}}\right]+\left(Q_{k+1}+\lambda_{k+1}^{i-1}\right)\right)\left[\frac{1}{d_{k-1}}-\frac{1}{d_{k}}\right]}{\left[\frac{1}{d_{k-1}}-\frac{1}{d_{k+1}}\right]} \\
& \Longrightarrow Q_{k}<\frac{Q_{v}\left[\frac{1}{d_{k}}-\frac{1}{d_{k+1}}\right]+Q_{k+1}\left[\frac{1}{d_{v}}-\frac{1}{d_{k}}\right]}{\left[\frac{1}{d_{v}}-\frac{1}{d_{k+1}}\right]} .
\end{aligned}
$$

Now, note that from (13), for every $n=v+1, \ldots, k$

$$
\lambda_{n}^{i}=\frac{Q_{v}\left[\frac{1}{d_{n}}-\frac{1}{d_{k+1}}\right]+Q_{k+1}\left[\frac{1}{d_{v}}-\frac{1}{d_{n}}\right]}{\left[\frac{1}{d_{v}}-\frac{1}{d_{k+1}}\right]}-Q_{n} .
$$

From (21) and (22), clearly, $\lambda_{k}^{i}>0$. Now, from (20) and (22), it follows that for every $n=v+$ $1, \ldots, k-1$

$$
\begin{aligned}
\lambda_{n}^{i}-\lambda_{n}^{i-1} & =\frac{Q_{v}\left[\frac{1}{d_{n}}-\frac{1}{d_{k+1}}\right]+Q_{k+1}\left[\frac{1}{d_{v}}-\frac{1}{d_{n}}\right]}{\left[\frac{1}{d_{v}}-\frac{1}{d_{k+1}}\right]}-\frac{Q_{v}\left[\frac{1}{d_{n}}-\frac{1}{d_{k}}\right]+Q_{k}\left[\frac{1}{d_{v}}-\frac{1}{d_{n}}\right]}{\left[\frac{1}{d_{v}}-\frac{1}{d_{k}}\right]} \\
& =\frac{\left[\frac{1}{d_{v}}-\frac{1}{d_{n}}\right]}{\left[\frac{1}{d_{v}}-\frac{1}{d_{k}}\right]} \lambda_{k}^{i} \geq 0 .
\end{aligned}
$$

The last inequality follows from the fact that $d_{n} \leq d_{n+1}$ for every $n$ and $\lambda_{k}^{i}>0$. Furthermore, by Lemma 3, for $n \notin\{v+1, \ldots, k\}, \lambda_{n}^{i-1}=\lambda_{n}^{i}$. Thus, the result follows.

Lemma 7: Let index $k>1$ be added to the set $\mathcal{E}^{i-1}$ in the $i^{\text {th }}$ iteration. Also, let $\{1, \ldots, k-1\} \subseteq$ $\mathcal{E}^{i-1}$ and $k+1 \in \mathcal{A}^{i}$. Then, $\lambda_{n}^{i}-\lambda_{n}^{i-1} \geq 0$ for every $n$.

Proof: Since index $k$ is added to $\mathcal{E}^{i-1}$ in the $i^{\text {th }}$ iteration, we know the following. First, $\lambda_{k}^{i-1}=0$. Second, from (10)

$$
Q_{k}<\frac{\left.\left(Q_{k-1}+\lambda_{k-1}^{i-1}\right)\left[\frac{1}{d_{k}}-\frac{1}{d_{k+1}}\right]+\left(Q_{k+1}+\lambda_{k+1}^{i-1}\right)\right)\left[\frac{1}{d_{k-1}}-\frac{1}{d_{k}}\right]}{\left[\frac{1}{d_{k-1}}-\frac{1}{d_{k+1}}\right]} .
$$

Moreover, since $\{1, \ldots, k-1\} \subseteq \mathcal{E}^{i-1}$ and $k \in \mathcal{A}^{i-1}$, we also know that for every $n \in\{1, \ldots, k-$ $1\}$,

$$
\lambda_{n}^{i-1}=V N_{0}\left[\frac{1}{d_{n}}-\frac{1}{d_{k}}\right]+\left(Q_{k}-Q_{n}\right) .
$$

Now, substituting $\lambda_{k+1}^{i-1}=0$ and $\lambda_{k-1}^{i-1}$ from (24) in (23), we obtain

$$
\begin{aligned}
Q_{k} & <\frac{\left(V N_{0}\left[\frac{1}{d_{k-1}}-\frac{1}{d_{k}}\right]+Q_{k}\right)\left[\frac{1}{d_{k}}-\frac{1}{d_{k+1}}\right]+Q_{k+1}\left[\frac{1}{d_{k-1}}-\frac{1}{d_{k}}\right]}{\left[\frac{1}{d_{k-1}}-\frac{1}{d_{k+1}}\right]} \\
\Longrightarrow Q_{k} & <V N_{0}\left[\frac{1}{d_{k}}-\frac{1}{d_{k+1}}\right]+Q_{k+1} .
\end{aligned}
$$


Now, note that from (13), for every $n=1, \ldots, k$

$$
\lambda_{n}^{i}=V N_{0}\left[\frac{1}{d_{n}}-\frac{1}{d_{k+1}}\right]+\left(Q_{k+1}-Q_{n}\right) .
$$

From (26) and (27), clearly, $\lambda_{k}^{i}>0$. Now, from (24) and (27), it follows that for every $n=1, \ldots, k-1$

$$
\begin{aligned}
\lambda_{n}^{i}-\lambda_{n}^{i-1} & =V N_{0}\left[\frac{1}{d_{k}}-\frac{1}{d_{k+1}}\right]+\left(Q_{k+1}-Q_{k}\right) \\
& =\lambda_{k}^{i} \geq 0 .
\end{aligned}
$$

Furthermore, by Lemma 3, for $n \notin\{1, \ldots, k\}, \lambda_{n}^{i-1}=\lambda_{n}^{i}$. Thus, the result follows.

Lemma 8: Let index $k>1$ be added to the set $\mathcal{E}^{i-1}$ in the $i^{\text {th }}$ iteration. Also, let $\{k+1, \ldots, u-1\} \subseteq$ $\mathcal{E}^{i-1}$ and $\{k-1, u\} \subseteq \mathcal{A}^{i}$. Then, $\lambda_{n}^{i}-\lambda_{n}^{i-1} \geq 0$ for every $n$.

Proof: Since index $k$ is added to $\mathcal{E}^{i-1}$ in the $i^{\text {th }}$ iteration, we know the following. First, $\lambda_{k}^{i-1}=0$. Second, from (10)

$$
Q_{k}<\frac{\left.\left(Q_{k-1}+\lambda_{k-1}^{i-1}\right)\left[\frac{1}{d_{k}}-\frac{1}{d_{k+1}}\right]+\left(Q_{k+1}+\lambda_{k+1}^{i-1}\right)\right)\left[\frac{1}{d_{k-1}}-\frac{1}{d_{k}}\right]}{\left[\frac{1}{d_{k-1}}-\frac{1}{d_{k+1}}\right]} .
$$

Moreover, since $\{k+1, \ldots, u-1\} \subseteq \mathcal{E}^{i-1}$ and $\{k-1, k, u\} \subseteq \mathcal{A}^{i-1}$, we also know that for every $n \in\{k+1, \ldots, u-1\}$,

$$
\lambda_{n}^{i-1}=\frac{Q_{k}\left[\frac{1}{d_{n}}-\frac{1}{d_{u}}\right]+Q_{u}\left[\frac{1}{d_{k}}-\frac{1}{d_{n}}\right]}{\left[\frac{1}{d_{k}}-\frac{1}{d_{u}}\right]}-Q_{n} .
$$

Now, substituting $\lambda_{k-1}^{i-1}=0$ and $\lambda_{k+1}^{i-1}$ from (29) in (28), we obtain

$$
\begin{aligned}
Q_{k} & <\frac{Q_{k-1}\left[\frac{1}{d_{k}}-\frac{1}{d_{k+1}}\right]+\frac{Q_{k}\left[\frac{1}{d_{k+1}}-\frac{1}{d_{u}}\right]+Q_{u}\left[\frac{1}{d_{k}}-\frac{1}{d_{k+1}}\right]}{\left[\frac{1}{d_{k}}-\frac{1}{d_{u}}\right]}\left[\frac{1}{d_{k-1}}-\frac{1}{d_{k}}\right]}{\left[\frac{1}{d_{k-1}}-\frac{1}{d_{k+1}}\right]} \\
\Longrightarrow Q_{k} & <\frac{Q_{k-1}\left[\frac{1}{d_{k}}-\frac{1}{d_{u}}\right]+Q_{u}\left[\frac{1}{d_{k-1}}-\frac{1}{d_{k}}\right]}{\left[\frac{1}{d_{k-1}}-\frac{1}{d_{u}}\right]} .
\end{aligned}
$$

Now, note that from (13), for every $n=k, \ldots, u-1$

$$
\lambda_{n}^{i}=\frac{Q_{k-1}\left[\frac{1}{d_{n}}-\frac{1}{d_{u}}\right]+Q_{u}\left[\frac{1}{d_{k-1}}-\frac{1}{d_{n}}\right]}{\left[\frac{1}{d_{k-1}}-\frac{1}{d_{u}}\right]}-Q_{n} .
$$

From (30) and (31), clearly, $\lambda_{k}^{i}>0$. Now, from (29) and (31), it follows that for every $n=k+$ $1, \ldots, u-1$

$$
\begin{aligned}
\lambda_{n}^{i}-\lambda_{n}^{i-1} & =\frac{Q_{k-1}\left[\frac{1}{d_{n}}-\frac{1}{d_{u}}\right]+Q_{u}\left[\frac{1}{d_{k-1}}-\frac{1}{d_{n}}\right]}{\left[\frac{1}{d_{k-1}}-\frac{1}{d_{u}}\right]}-\frac{Q_{k}\left[\frac{1}{d_{n}}-\frac{1}{d_{u}}\right]+Q_{u}\left[\frac{1}{d_{k}}-\frac{1}{d_{n}}\right]}{\left[\frac{1}{d_{k}}-\frac{1}{d_{u}}\right]} \\
& =\frac{\left[\frac{1}{d_{n}}-\frac{1}{d_{u}}\right]}{\left[\frac{1}{d_{k}}-\frac{1}{d_{u}}\right]} \lambda_{k}^{i} \geq 0 .
\end{aligned}
$$


The last inequality follows from the fact that $d_{n} \leq d_{n+1}$ for every $n$ and $\lambda_{k}^{i}>0$. Furthermore, by Lemma 3, for $n \notin\{k, \ldots, u-1\}, \lambda_{n}^{i-1}=\lambda_{n}^{i}$. Thus, the result follows.

Lemma 9: Let index $k>1$ be added to the set $\mathcal{E}^{i-1}$ in the $i^{\text {th }}$ iteration. Also, let $\{v+1, \ldots, k-$ $1\} \cup\{k+1, \ldots, u-1\} \subseteq \mathcal{E}^{i-1}$ and $\{v, u\} \subseteq \mathcal{A}^{i}$. Then, $\lambda_{n}^{i}-\lambda_{n}^{i-1} \geq 0$ for every $n$.

Proof: Since index $k$ is added to $\mathcal{E}^{i-1}$ in the $i^{\text {th }}$ iteration, we know the following. First, $\lambda_{k}^{i-1}=0$. Second, from (10)

$$
Q_{k}<\frac{\left.\left(Q_{k-1}+\lambda_{k-1}^{i-1}\right)\left[\frac{1}{d_{k}}-\frac{1}{d_{k+1}}\right]+\left(Q_{k+1}+\lambda_{k+1}^{i-1}\right)\right)\left[\frac{1}{d_{k-1}}-\frac{1}{d_{k}}\right]}{\left[\frac{1}{d_{k-1}}-\frac{1}{d_{k+1}}\right]} .
$$

Moreover, since $\{v+1, \ldots, k-1\} \cup\{k+1, \ldots, u-1\} \subseteq \mathcal{E}^{i-1}$ and $\{v, k, u\} \subseteq \mathcal{A}^{i-1}$, we also know that for every $n \in\{v+1, \ldots, k-1\}$,

$$
\lambda_{n}^{i-1}=\frac{Q_{v}\left[\frac{1}{d_{n}}-\frac{1}{d_{k}}\right]+Q_{k}\left[\frac{1}{d_{v}}-\frac{1}{d_{n}}\right]}{\left[\frac{1}{d_{v}}-\frac{1}{d_{k}}\right]}-Q_{n},
$$

and for every $n \in\{k+1, \ldots, u-1\}$,

$$
\lambda_{n}^{i-1}=\frac{Q_{k}\left[\frac{1}{d_{n}}-\frac{1}{d_{u}}\right]+Q_{u}\left[\frac{1}{d_{k}}-\frac{1}{d_{n}}\right]}{\left[\frac{1}{d_{k}}-\frac{1}{d_{u}}\right]}-Q_{n} .
$$

Now, substituting $\lambda_{k-1}^{i-1}$ and $\lambda_{k+1}^{i-1}$ from (33) and (34), respectively, in (32), we obtain

$$
\begin{aligned}
& Q_{k}< \frac{\frac{Q_{v}\left[\frac{1}{d_{k-1}}-\frac{1}{d_{k}}\right]+Q_{k}\left[\frac{1}{d_{v}}-\frac{1}{d_{k-1}}\right]}{\left[\frac{1}{d_{v}}-\frac{1}{d_{k}}\right]}\left[\frac{1}{d_{k}}-\frac{1}{d_{k+1}}\right]+\frac{Q_{k}\left[\frac{1}{d_{k+1}}-\frac{1}{d_{u}}\right]+Q_{u}\left[\frac{1}{d_{k}}-\frac{1}{d_{k+1}}\right]}{\left[\frac{1}{d_{k}}-\frac{1}{d_{u}}\right]}\left[\frac{1}{d_{k-1}}-\frac{1}{d_{k}}\right]}{\left[\frac{1}{d_{k-1}}-\frac{1}{d_{k+1}}\right]} \\
& \Longrightarrow Q_{k}<\frac{Q_{v}\left[\frac{1}{d_{k}}-\frac{1}{d_{u}}\right]+Q_{u}\left[\frac{1}{d_{v}}-\frac{1}{d_{k}}\right]}{\left[\frac{1}{d_{v}}-\frac{1}{d_{u}}\right]} .
\end{aligned}
$$

Now, note that from (13), for every $n=v+1, \ldots, u-1$

$$
\lambda_{n}^{i}=\frac{Q_{v}\left[\frac{1}{d_{n}}-\frac{1}{d_{u}}\right]+Q_{u}\left[\frac{1}{d_{v}}-\frac{1}{d_{n}}\right]}{\left[\frac{1}{d_{v}}-\frac{1}{d_{u}}\right]}-Q_{n} .
$$

From (35) and (36), clearly, $\lambda_{k}^{i}>0$. Now, from (33) and (36), it follows that for every $n=v+$ $1, \ldots, k-1$

$$
\begin{aligned}
\lambda_{n}^{i}-\lambda_{n}^{i-1} & =\frac{Q_{v}\left[\frac{1}{d_{n}}-\frac{1}{d_{u}}\right]+Q_{u}\left[\frac{1}{d_{v}}-\frac{1}{d_{n}}\right]}{\left[\frac{1}{d_{v}}-\frac{1}{d_{u}}\right]}-\frac{Q_{v}\left[\frac{1}{d_{n}}-\frac{1}{d_{k}}\right]+Q_{k}\left[\frac{1}{d_{v}}-\frac{1}{d_{n}}\right]}{\left[\frac{1}{d_{v}}-\frac{1}{d_{k}}\right]} \\
& =\frac{\left[\frac{1}{d_{v}}-\frac{1}{d_{n}}\right]}{\left[\frac{1}{d_{v}}-\frac{1}{d_{k}}\right]} \lambda_{k}^{i} \geq 0 .
\end{aligned}
$$


The last inequality follows from the fact that $d_{n} \leq d_{n+1}$ for every $n$ and $\lambda_{k}^{i}>0$. Moreover, from (34) and (36), it follows that for every $n=k+1, \ldots, u-1$

$$
\begin{aligned}
\lambda_{n}^{i}-\lambda_{n}^{i-1} & =\frac{Q_{v}\left[\frac{1}{d_{n}}-\frac{1}{d_{u}}\right]+Q_{u}\left[\frac{1}{d_{v}}-\frac{1}{d_{n}}\right]}{\left[\frac{1}{d_{v}}-\frac{1}{d_{u}}\right]}-\frac{Q_{k}\left[\frac{1}{d_{n}}-\frac{1}{d_{u}}\right]+Q_{u}\left[\frac{1}{d_{k}}-\frac{1}{d_{n}}\right]}{\left[\frac{1}{d_{k}}-\frac{1}{d_{u}}\right]} \\
& =\frac{\left[\frac{1}{d_{n}}-\frac{1}{d_{u}}\right]}{\left[\frac{1}{d_{k}}-\frac{1}{d_{u}}\right]} \lambda_{k}^{i} \geq 0 .
\end{aligned}
$$

Furthermore, by Lemma 3, for $n \notin\{v+1, \ldots, u-1\}, \lambda_{n}^{i-1}=\lambda_{n}^{i}$. Thus, the result follows.

Lemma 10: Let index $k>1$ be added to the set $\mathcal{E}^{i-1}$ in the $i^{\text {th }}$ iteration. Also, let $\{1, \ldots, k-$ $1\} \cup\{k+1, \ldots, u-1\} \subseteq \mathcal{E}^{i-1}$ and $u \in \mathcal{A}^{i}$. Then, $\lambda_{n}^{i}-\lambda_{n}^{i-1} \geq 0$ for every $n$.

Proof: Since index $k$ is added to $\mathcal{E}^{i-1}$ in the $i^{\text {th }}$ iteration, we know the following. First, $\lambda_{k}^{i-1}=0$. Second, from (10)

$$
Q_{k}<\frac{\left.\left(Q_{k-1}+\lambda_{k-1}^{i-1}\right)\left[\frac{1}{d_{k}}-\frac{1}{d_{k+1}}\right]+\left(Q_{k+1}+\lambda_{k+1}^{i-1}\right)\right)\left[\frac{1}{d_{k-1}}-\frac{1}{d_{k}}\right]}{\left[\frac{1}{d_{k-1}}-\frac{1}{d_{k+1}}\right]} .
$$

Moreover, since $\{1, \ldots, k-1\} \cup\{k+1, \ldots, u-1\} \subseteq \mathcal{E}^{i-1}$ and $\{k, u\} \subseteq \mathcal{A}^{i-1}$, we also know that for every $n \in\{1, \ldots, k-1\}$,

$$
\lambda_{n}^{i-1}=V N_{0}\left[\frac{1}{d_{n}}-\frac{1}{d_{k}}\right]+\left(Q_{k}-Q_{n}\right)
$$

and for every $n \in\{k+1, \ldots, u-1\}$,

$$
\lambda_{n}^{i-1}=\frac{Q_{k}\left[\frac{1}{d_{n}}-\frac{1}{d_{u}}\right]+Q_{u}\left[\frac{1}{d_{k}}-\frac{1}{d_{n}}\right]}{\left[\frac{1}{d_{k}}-\frac{1}{d_{u}}\right]}-Q_{n} .
$$

Now, substituting $\lambda_{k-1}^{i-1}$ and $\lambda_{k+1}^{i-1}$ from (38) and (39), respectively, in (37), we obtain

$$
\begin{aligned}
Q_{k} & <\frac{\left[V N_{0}\left[\frac{1}{d_{k-1}}-\frac{1}{d_{k}}\right]+Q_{k}\right]\left[\frac{1}{d_{k}}-\frac{1}{d_{k+1}}\right]+\frac{Q_{k}\left[\frac{1}{d_{k+1}}-\frac{1}{d_{u}}\right]+Q_{u}\left[\frac{1}{d_{k}}-\frac{1}{d_{k+1}}\right]}{\left[\frac{1}{d_{k}}-\frac{1}{d_{u}}\right]}\left[\frac{1}{d_{k-1}}-\frac{1}{d_{k}}\right]}{\left[\frac{1}{d_{k-1}}-\frac{1}{d_{k+1}}\right]} \\
\Longrightarrow Q_{k} & <V N_{0}\left[\frac{1}{d_{k}}-\frac{1}{d_{u}}\right]+Q_{u} .
\end{aligned}
$$

Now, note that from (13), for every $n=1, \ldots, u-1$

$$
\lambda_{n}^{i}=V N_{0}\left[\frac{1}{d_{n}}-\frac{1}{d_{u}}\right]+\left(Q_{u}-Q_{n}\right) .
$$

From (40) and (41), clearly, $\lambda_{k}^{i}>0$. Now, from (38) and (41), it follows that for every $n=1, \ldots, k-1$

$$
\begin{aligned}
\lambda_{n}^{i}-\lambda_{n}^{i-1} & =V N_{0}\left[\frac{1}{d_{n}}-\frac{1}{d_{u}}\right]+\left(Q_{u}-Q_{n}\right)-V N_{0}\left[\frac{1}{d_{n}}-\frac{1}{d_{k}}\right]-\left(Q_{k}-Q_{n}\right) \\
& =\lambda_{k}^{i} \geq 0 .
\end{aligned}
$$


The last inequality follows from the fact that and $\lambda_{k}^{i}>0$. Moreover, from (39) and (41), it follows that for every $n=k+1, \ldots, u-1$

$$
\begin{aligned}
\lambda_{n}^{i}-\lambda_{n}^{i-1} & =V N_{0}\left[\frac{1}{d_{n}}-\frac{1}{d_{u}}\right]+Q_{u}-\frac{Q_{k}\left[\frac{1}{d_{n}}-\frac{1}{d_{u}}\right]+Q_{u}\left[\frac{1}{d_{k}}-\frac{1}{d_{n}}\right]}{\left[\frac{1}{d_{k}}-\frac{1}{d_{u}}\right]} \\
& =\left[\frac{1}{d_{n}}-\frac{1}{d_{u}}\right] \lambda_{k}^{i} \geq 0 .
\end{aligned}
$$

Furthermore, by Lemma 3, for $n \notin\{1, \ldots, u-1\}, \lambda_{n}^{i-1}=\lambda_{n}^{i}$. Thus, the result follows. 